\title{
Image Segmentation and 3D reconstruction for improved prediction of the sublimation rate during freeze drying
}

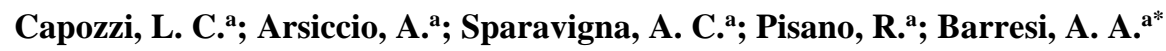 \\ a Department of Applied Science and Technology, Politecnico di Torino, Torino, Italy \\ *E-mail of the corresponding author: antonello.barresi@polito.it
}

\begin{abstract}
In a freeze drying process, the freezing step determines the pore size distribution within the product, which, in turn, affects the sublimation rate. Traditionally, pore analysis is carried out on SEM images by means of a manual, time-consuming approach. Here, an image segmentation technique was used to automatize this process and improve its reliability. A $3 D$ structure of the cake was then reconstructed from the distribution of the super-pixels. We show that the approach herein proposed can remarkably improve prediction of the sublimation rate with respect to traditional methods.
\end{abstract}

Keywords: Freezing; Freeze-Drying; Image Segmentation; 3D Reconstruction 


\section{Introduction}

During freeze drying, water is removed by sublimation and desorption from a previously frozen product. When designing a freeze drying process, it is well-known that the freezing step is extremely relevant for both process performance and product quality [1]. In fact, different freezing protocols result in different pore size distributions within the product, affecting both sublimation and desorption rates. More specifically, the nucleation event, and, in particular, the temperature at which the first ice crystal nuclei are formed, is of major importance in the freezing process, as it determines the pore size distribution which will then be formed in the dried sample. In particular, a low nucleation temperature will result in the formation of small pores. These small pores will then offer a large resistance to water vapor flow during primary drying, thus decreasing the sublimation rate. From this viewpoint, a problem with the traditional freezing approaches, such as shelf ramped freezing, is that they do not allow control of the nucleation temperature, which will therefore be stochastically distributed. However, some techniques have been developed to overcome this problem [1,2]. Among them, vacuum induced surface freezing (VISF) was demonstrated to be beneficial for both product homogeneity and process performance [3,4]. In fact, VISF can be used to induce nucleation at a higher temperature in all the vials being processed. This higher nucleation temperature results in the formation of larger pores within the dried samples, boosting the sublimation rate. For instance, in Figure 1 two Scanning Electron Microscopy (SEM) images are shown, representing a 5\% w/w mannitol sample after freeze drying. In the case of Figure 1a, VISF was used, while the sample in Figure $1 \mathrm{~b}$ was processed using the conventional shelf-ramped freezing protocol.

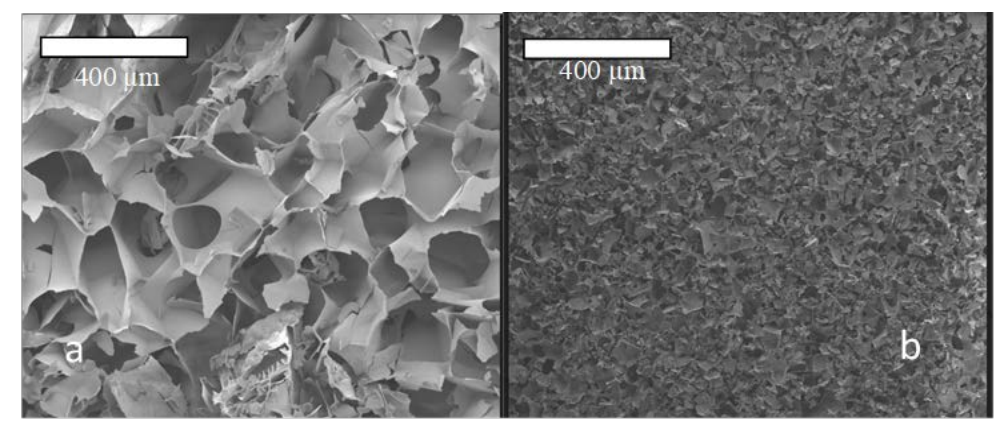

Fig. 1 SEM images of 5\% w/w mannitol samples obtained using VISF (a) or shelf ramped freezing (b).

The huge difference in sample morphology which can be observed translates into remarkably different cycle performance; it is therefore clear that the information on the pore size distribution is crucial for the proper design of a freeze-drying process. Although many other 
approaches have been proposed in the past, such as X-ray tomography [5], the most common technique remains off-line SEM analysis. This is generally achieved by means of a manual analysis of the resulting images. The manual approach is, however, highly time consuming, and the results obtained are subjective and, therefore, poorly reliable. Therefore, we recently proposed a method based on the segmentation of SEM images to automatically determine the pore size distribution [6]. In the above-mentioned work, we showed that image segmentation could quickly and reliably allow resolution of the porous structure of the sample under investigation. Here we propose a further improvement of this technique, which combines the analysis of the levels of grey-tones to the detection of edges between different domains. Moreover, we will show that, from the super-pixels distribution obtained as a result of segmentation, a 3D structure of the cake may be reconstructed by means of a stochastic approach. We will prove that the reconstruction obtained can fairly well represent the main features of the real dried cake. This result is important, since the generated structure may then be used within a computational fluid dynamics (CFD) simulation, to estimate the tortuosity of the sample [7]. Since both the pore size distribution and the tortuosity factor enter into the definition of the mass transfer resistance to vapor flow, the approach herein proposed could remarkably improve prediction of the sublimation rate, which is at the basis of any model for the freeze-drying process.

\section{Materials and Methods}

\subsection{Freeze drying cycles and SEM analysis}

The formulation investigated in this work was a 5\% w/w mannitol solution, prepared from distilled water and mannitol powder (Sigma Aldrich). The vials employed (type 1, 10R, 45x24 mm, Schott AG, Germany), where filled in with $3 \mathrm{ml}$ of sample solution, and loaded onto the shelves of a freeze-dryer LyoBeta 25 (Telstar, Terrassa, Spain). For the conventional (shelf ramped) freezing tests, the refrigerating fluid temperature was decreased to $-45^{\circ} \mathrm{C}$ at $0.7^{\circ} \mathrm{C} / \mathrm{min}$.

As regards VISF, the product was first equilibrated at $-5^{\circ} \mathrm{C}$ for $45 \mathrm{~min}$ and, then, the chamber pressure was reduced to $130 \mathrm{~Pa}$ until nucleation was observed. The pressure was then quickly released to the atmospheric value, and the product was equilibrated at $-10^{\circ} \mathrm{C}$ for $1 \mathrm{~h}$. To complete the freezing of the product, the refrigerating fluid temperature was subsequently decreased to $-45^{\circ} \mathrm{C}$ at $0.7^{\circ} \mathrm{C} / \mathrm{min}$ and held for $1 \mathrm{~h}$. Finally, drying was performed at $10 \mathrm{~Pa}$, and $-10^{\circ} \mathrm{C}$. The internal structure of the resulting cake was analyzed by means of a Scanning Electron Microscope (SEM, FEI type, Quanta Inspect 200, Eindhoven, the Netherlands). 


\subsection{Image Segmentation for the processing of SEM images}

In [6], we described a segmentation approach, based on the analysis of the levels of greytones, for the determination of the pore size distribution in freeze dried samples. Here, we propose an improved version of this technique, in which the detection of the edges between the pores and the surrounding scaffold is included in the analysis. First, the SEM image is processed to enhance the edges in it, that is processed to detect the regions where we have a large gradient in the topography. Among the methods for edge detection, the Retinex filter, which is available in GIMP (the GNU Image Manipulation Program [8]), was chosen. Then, this information is merged to that concerning the topography itself and the image converted into a binary black and white map (Figures 2a and 2b). From the resulting map, the pores are automatically selected, as described in [6], and as shown in Figures 2c and 2d.

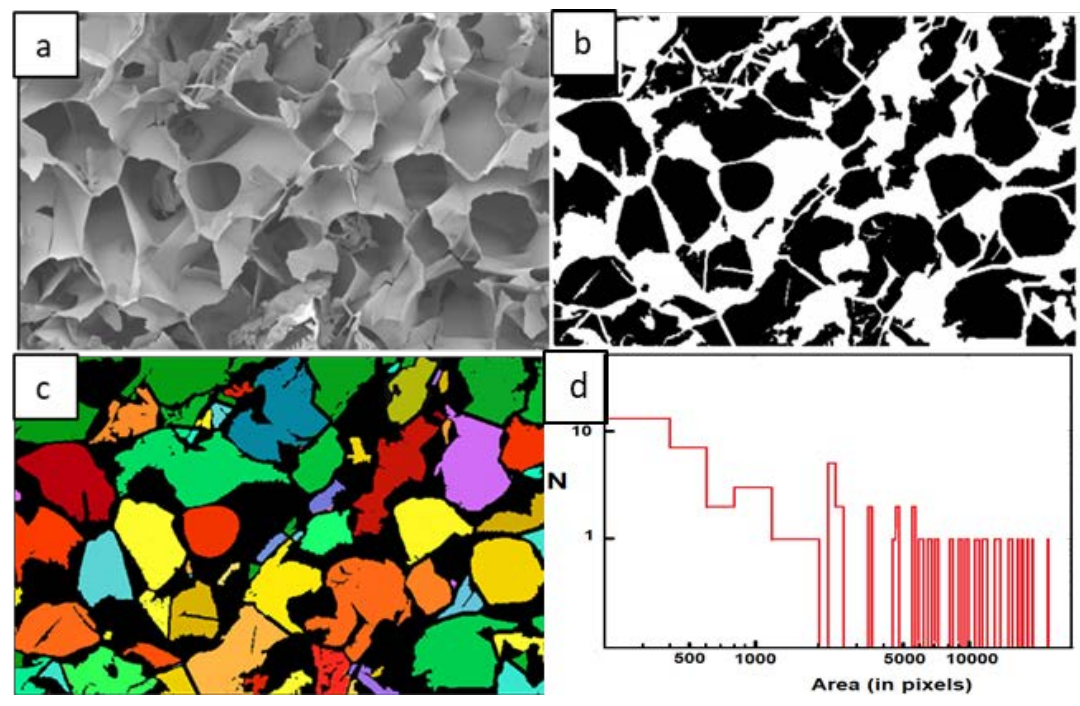

Fig. 2 (a) SEM and (b) binary images corresponding to mannitol 5\% $w / w$, freeze dried using VISF. (c) Segmentation of the original SEM image, where the domains are represented by different colors. (d) Distribution of the super-pixels (in logarithmic scales), obtained by counting them according to their area (in pixels).

\subsection{Prediction of the resistance to mass transfer}

The resistance to mass transfer, $R_{p}$, was calculated from the average pore dimension $D_{p}$, as calculated using either the manual approach, or the image segmentation technique, by means of the following equation, 


$$
R_{p}=\frac{3}{2} \frac{\tau^{2}}{\varepsilon} \frac{L_{d}}{D_{p}} \sqrt{\frac{\pi \mathrm{R} T}{2 M_{w}}}
$$

where $\mathrm{R}$ and $M_{w}$ are the universal gas constant and water molecular weight, respectively. As can be seen, $R_{p}$ is a function of the dried layer thickness $L_{d}$, and depends on the product temperature $T$, and on the dried product tortuosity factor $\tau$ and porosity $\varepsilon$, as well.

\subsection{Stochastic reconstruction of 3D structures}

Using the information obtained from image analysis, a 3D structure of the dried cake was then reconstructed, for the case of the product obtained by VISF (Figure 1a). To do this, spherical pores were stochastically built into an 800 x 800 x $800 \mu \mathrm{m}$ original bulky cube. The dimension of the spheres was chosen so as to reproduce the pore size distribution obtained from image segmentation (Figure 2d). As shown in Figure 3 this procedure was iterated until the desired value of porosity was reached. No constraints were imposed on the position of the sphere centers, so that interpenetration of the pores was possible, as illustrated in Figure 3. This was done in order to better reproduce the real structure of a freeze-dried product, where the porous structure is, generally, highly interconnected.
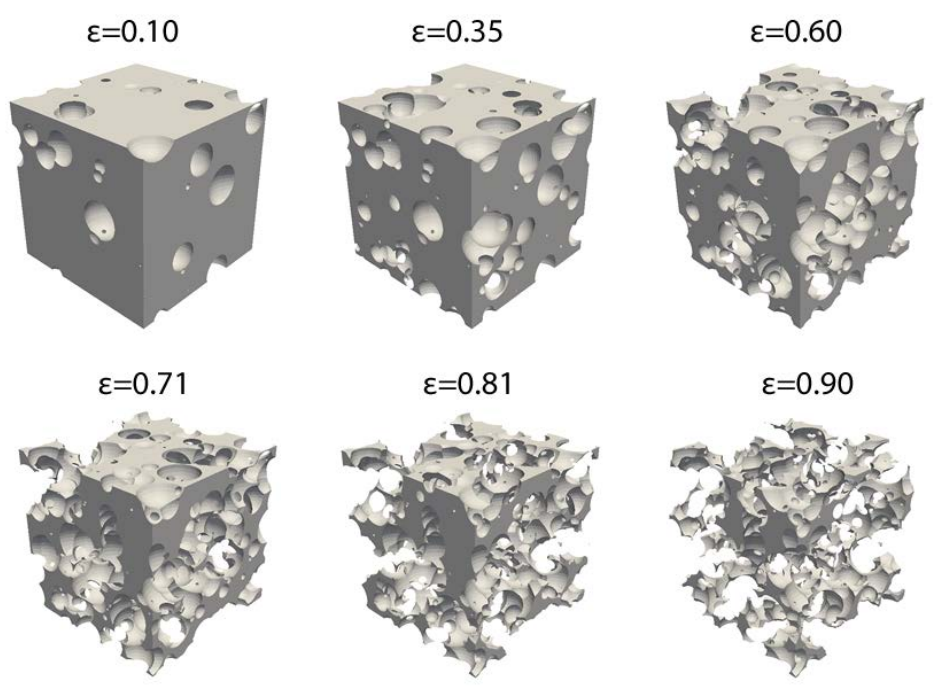

Fig. 3 Reconstruction of a lyophilized cake with different void fraction. 


\section{Results and Discussion}

The image segmentation technique described in section 2.2 was applied to the SEM images shown in Figure 1. As illustrated in a previous work [6], this technique allows calculation of the super-pixels distribution within the SEM image, from which an average pore size can be extracted. For instance, using segmentation, average pore dimensions of $140 \pm 30 \mu \mathrm{m}$ and $36 \pm 6 \mu \mathrm{m}$ were obtained for VISF (Figure 1a) and shelf ramped freezing (Figure 1b), respectively. By contrast, using the traditional approach, that is, manually selecting and measuring a sufficiently large number of pores, the conventional freezing protocol and VISF resulted in an average diameter of $20 \pm 8 \mu \mathrm{m}$ and $120 \pm 36 \mu \mathrm{m}$, respectively. As can be seen, both techniques measured a larger average pore size in the case of VISF, which was expected, because of the higher nucleation temperature in the case of controlled nucleation. However, the pore dimension is not sufficient for an accurate estimation of the resistance to mass transfer $R_{p}$, since another required parameter is the tortuosity factor $\tau$, whose value is generally only roughly hypothesized. Typically used values span between 1.2 and 3.8 [9], and this range translates into a large uncertainty in the value of $R_{p}$, as shown in Figure 4.
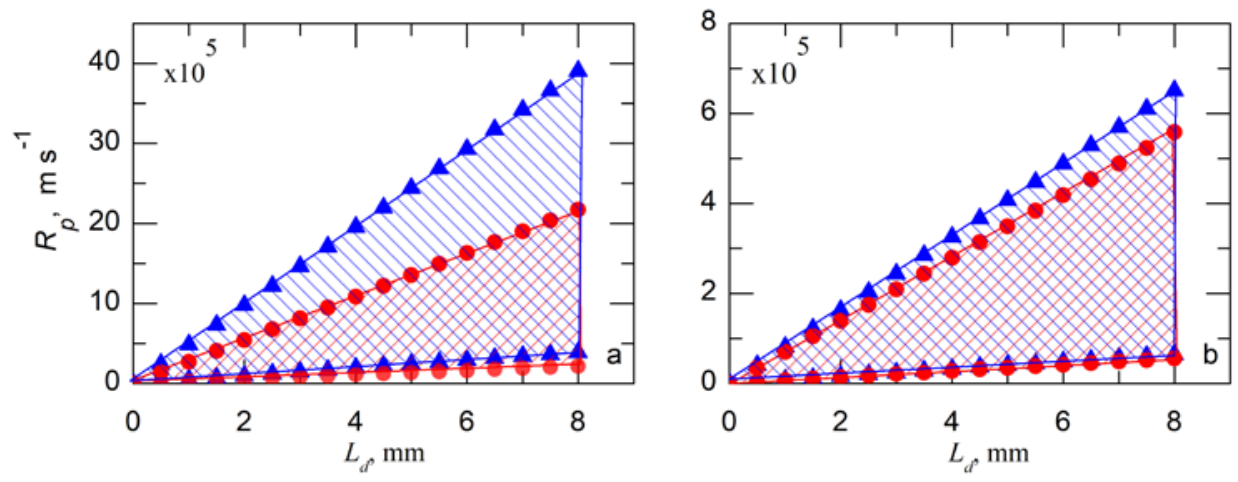

Fig. 4 Uncertainty in the value of $R_{p}$ as function of the dried layer thickness $L_{d}$, due to the uncertainty on $\tau$. The range 1.2-3.8 was considered for $\tau$. (a) conventional freezing, and (b) VISF. $\bullet:$ Values calculated using Eq. 1, in which the pore dimension $D_{p}$ was obtained by the image segmentation approach; $\Delta$ : Values calculated using Eq. 1, in which the pore dimension $D_{p}$ was obtained by the traditional manual approach.

A possible solution to this problem can be found combining image analysis and CFD simulations. In fact, as described in section 2.4, a 3D structure of the dried cake might be reconstructed using the super-pixels distribution obtained from image segmentation. An example of reconstructed structure, for varying values of porosity, is shown in Figure 3. The stochastic approach used for the $3 \mathrm{D}$ reconstruction was validated against the original SEM image, as outlined in Figure 5. A 800 × 800 x $400 \mu \mathrm{m}$ slice was isolated from the reconstructed cube, and a 2D section (Figure $5 b$ ) was obtained from this slice, using a grey- 
tone scale ranging from white to black to represent perspective. This was done in order to simulate the same type of representation obtained in a typical SEM analysis. As can be seen comparing Figures $5 \mathrm{a}$ and $5 \mathrm{~b}$, there was a striking similarity between the original SEM image and the 2D section obtained from the 3D reconstruction. To further confirm this similarity, Figure 5b was analyzed using image segmentation, and the super-pixels distribution was calculated (Figure 5c). Remarkably, the resulting distribution compares fairly well with that obtained for the original SEM image (Figure 2d). This means that the 3D reconstruction preserves most of the features of the original sample, and could therefore allow an easier analysis of the properties which influence the sublimation rate; for instance, by a CFD simulation of water vapor flow through the reconstructed 3D structure.
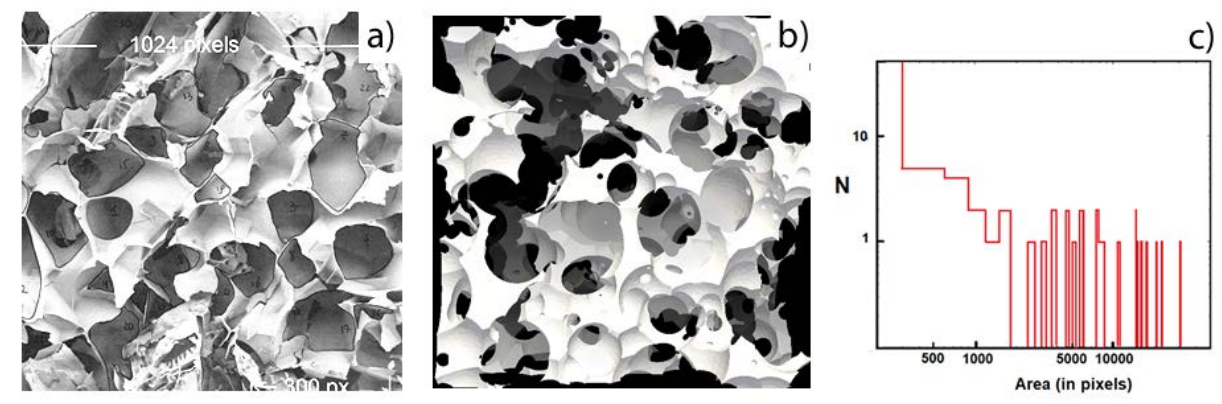

Fig. 5 Validation of the 3D reconstruction approach: a) SEM image, b) upper view of a slice of the $3 D$ structure and c) super-pixels distribution in Fig. $5 b$.

\section{Conclusions}

In the present work, a new approach has been proposed for the estimation of both the pore size distribution and the tortuosity factor within a freeze dried sample. Work is in progress to simulate the fluid dynamics in the 3D reconstructed matrix. Subsequently, analysis of the velocity vectors for the water vapor during the simulation would make it possible to estimate the tortuosity of the sample, and, thus, to calculate a more accurate value for the mass transfer resistance; the same procedure discussed in [7] for granular beds obtained by spray-freezing can be adopted. The described method may allow a significantly improved fitting of experimental data with respect to other traditional modeling techniques. We are therefore convinced that the approach herein outlined could be extremely beneficial, paving the way for a more robust design of the freeze drying process.

\section{Acknowledgements}

Computational resources were provided by ISCRA-Cineca HPC CLASS-C Grant to L.C.C. (ParticLy - HP10CQRVJV) 


\section{References}

[1] Kasper, J.C.; Friess, W.F. The freezing step in lyophilization: Physico-chemical fundamentals, freezing methods and consequences on process performance and quality attributes of biopharmaceuticals. European Journal of Pharmaceutics and Biopharmaceutics 2011, 78 (2), 248-263.

[2] Pisano, R.; Arsiccio, A.; Nakagawa, K.; Barresi, A.A. Control, measurement and prediction of the impact of freezing on product morphology: A step towards improved design of freeze-drying cycles. Drying Technology 2018, accepted.

[3] Oddone, I.; Pisano, R.; Bullich, R.; Stewart, P. Vacuum-induced nucleation as a method for freeze-drying cycle optimization. Industrial and Engineering Chemistry Research 2014, 53 (47), 18236-18244.

[4] Oddone, I.; Van Bockstal, P.-J.; De Beer, T.; Pisano, R. Impact of vacuum-induced surface freezing on inter- and intra-vial heterogeneity. European Journal of Pharmaceutics and Biopharmaceutics 2016, 103 (1), 167-178.

[5] Pisano, R.; Barresi, A.A.; Capozzi, L.C.; Novajra, G.; Oddone, I.; Vitale-Brovarone, C. Characterization of the mass transfer of lyophilized products based on X-ray microcomputed tomography images. Drying Technology 2017, 35 (8), 933-938.

[6] Arsiccio, A.; Sparavigna, A.C.; Pisano, R.; Barresi, A.A. Measuring and predicting pore size distribution of freeze-dried solutions. Drying Technology 2018, in press. DOI: 10.1080/07373937.2018.1430042

[7] Capozzi, L.C.; Boccardo, G.; Barresi, A.A.; Pisano, R. Computer-aided property estimation of micro-particles in packed-beds for freeze-drying applications. In Proceedings of $20^{\text {th }}$ International Drying Symposium, Gifu, Japan, August 7-10, 2016; paper A-4-3.

[8] Finlayson, G.D.; Hordley, S.D.; Drew, M.S. Removing shadows from images using retinex. In Proceedings of $10^{\text {th }}$ Color and Imaging Conference, Scottsdale, Arizona, January 2002; 73-79.

[9] Goshima, H.; Do, G.; Nakagawa, K. Impact of ice morphology on design space of pharmaceutical freeze-drying. Journal of Pharmaceutical Sciences 2016, 105 (6), 1920 1933. 